\title{
Effet de la date de labour sur l'état structural de l'horizon labouré dans les sols argileux gonflants du Marais poitevin
}

\author{
Sylvain PELLERIN $(*)$
}

I.N.R.A., Unité de recherche sur les Systèmes Agraires et le Développement, F 78850 Thiverval-Grignon

RÉSUMÉ

Dans les sols argileux gonflants du Marais poitevin la date de labour est a priori importante vis-à-vis de l'état structural créé dans l'horizon labouré. De cette date dépendent en effet :

- l'état hydrique du sol au moment du labour,

- les possibilités de fissuration ultérieures sous l'action des alternances d'humectation-dessiccation.

On a comparé l'état structural obtenu au moment des semis de maïs dans l'horizon labouré non repris pour les 3 traitements suivants :

- deux labours, notés L1 et $\mathrm{L} 2$, effectués en été sur sol sec, à même teneur en eau mais à des dates différentes, - un labour, noté $\mathbf{L} 3$, fait plus tard après réhumectation du profil.

Les traitements ont été classés par observation du profil cultural après semis et suivi de la culture (cartographie d'enracinement, mesures de croissance). Des observations intermédiaires entre labour et semis ont été réalisées pour suivre l'évolution des états structuraux.

Les résultats donnent, vis-à-vis de la qualité de l'état structural creé dans l'horizon labouré non repris, le classement suivant : $\mathrm{L} 1=\mathrm{L} 2>\mathrm{L} 3$. La fissuration, importante en surface (horizon 0-10 $\mathrm{cm}$ ), a peu concerné l'horizon labouré sous-jacent, même pour le labour le plus précoce, ce qui expliquerait l'absence d'effet date entre Ll et L2.

Tout se passe comme si l'avantage des labours précoces ( $\mathrm{L} 1$ et $\mathrm{L} 2$ ) par rapport à L3 était de créer par fragmentation une porosité extra-mottière qui autorise pendant l'hiver un gonflement des éléments structuraux sans contraintes, donc sans réduction de la porosité structurale intra-mottière.

Un calcul de bilan hydrique permet alors de placer très approximativement année par année les dates à partir desquelles l'état créé par le labour deviendrait défavorable car insuffisamment fragmenté.

Mots clés additionnels : Travail du sol, argiles gonflantes, porosité structurale, porosité texturale, mais.

Effect of ploughing date on the soil tilth of the ploughed horizon in swelling clay soils of "Marais poitevin" (France).

The effect of ploughing date was studied in swelling clay soils of "Marais poitevin" (France). Ploughing date is believed to be important because on it depend : 1) water content of soil, and consequently effect of ploughing; 2) cracking possibilities after ploughing, by alternate drying and swelling. We compared the soil tilth obtained in the part of the ploughed horizon which is not concerned by superficial tillage for the three following treatments : two treatments, called L1 and L2, where ploughing was done on dry soil, with the same water content but at different times ; a treatment, called $\mathrm{L} 3$, where ploughing was done after rehydration of the soil. The comparison was made by profile descriptions after sowing and investigations on the crop (root map and growth measurements). Other observations of profiles were done in order to follow soil tilth development. The results showed that soil tilth was the same in L1 and L2 and better than in L3.

Cracking affected only the soil surface $(0-10 \mathrm{~cm})$ and the ploughed horizon below seemed not to be concerned, even for the earlier ploughing (L1), possibly explaining why there was no difference between L1 and L2. The advantage of early ploughing (L1 and L2), compared to L3, seemed to be due to fragmentation. It created porosity between clods, allowing swelling during winter without decrease of structural porosity inside the clods.

Additional key words : Tillage, swelling clay soils, structural porosity, textural porosity, maize. 


\section{INTRODUCTION}

L'évolution récente de l'agriculture du Marais poitevin de Vendée a été marquée par un net accroissement des surfaces labourées. Or, les caractéristiques du milieu physique font du travail du sol une opération difficile et pourtant déterminante pour la réussite des cultures. En effet :

- Le climat est de type océanique, avec un fort excès d'eau hivernal et un déficit hydrique estival important (fig. 1). Les températures sont douces et les gelées sont rares.

- Les sols sont argileux, riches en smectites (MORIZET et al., 1970 ; NIJS, 1978), présentant de ce fait une forte aptitude à la fissuration mais aussi certaines propriétés physiques défavorables : faible conductivité hydraulique lorsqu'il y a fermeture par gonflement de la macroporosité fissurale, forte cohésion à l'état sec, plasticité et adhésivité élevées à l'état humide.

Il en résulte une période favorable d'intervention sur les parcelles, limitée dans le temps (problème de portance), et des difficultés pour obtenir une fragmentation par les outils (problèmes de plasticité-cohésion). Or l'état structural du profil conditionne, entre autres, l'implantation du système racinaire, donc les possibilités d'alimentation hydrique de la culture pendant la période de déficit (TARDIEU, 1984).

Les travaux effectués au Domaine I.N.R.A. de Saint-Laurent-de-la-Prée en matière de travail du sol

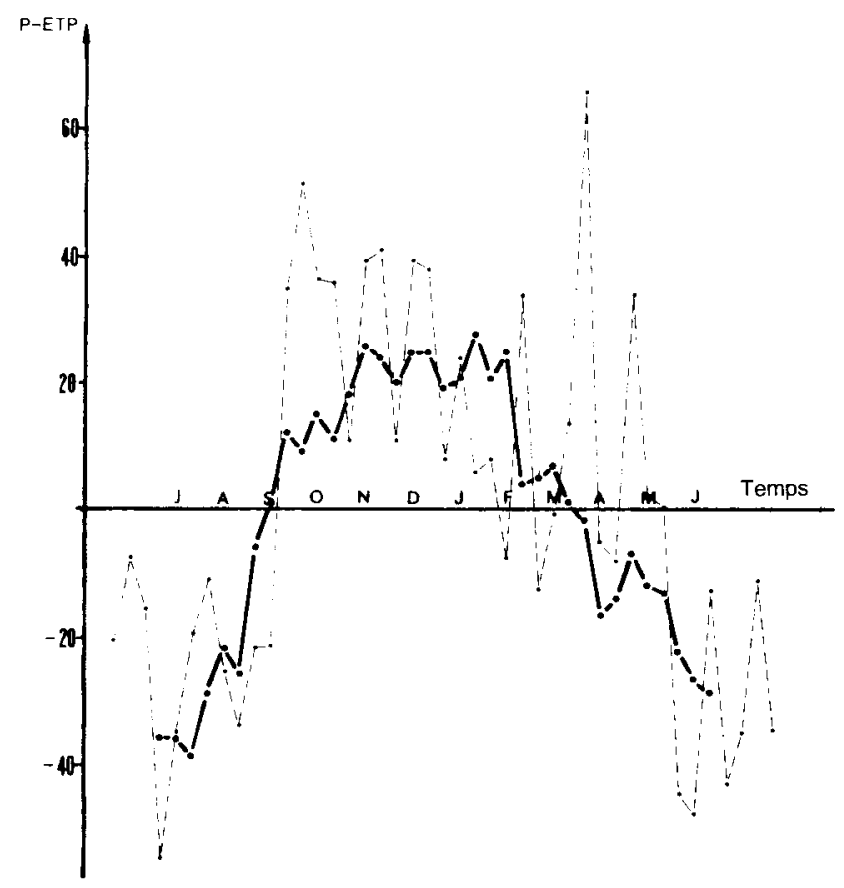

Figure 1

- P-ETP moyennes décadaires calculées sur la période 19671982 ; Mean 10-day values of P-ETP, calculated for the 1967-1982 period (P : rainfall ; ETP : potential evapotranspiration).

- - - P-ETP décadaires sur la période du 1/06/82 au 10/08/83 (Domaine INRA de St-Laurent-de-la-Prée, CharenteMaritime) ; 10-day values of P-ETP for the $1 / 06 / 82-$ 10/08/83 period. (Domaine INRA, St-Laurent-de-la-Prée, Charente-Maritime, France). ont abouti aux recommandations suivantes (DAMOUR \& NiCOUlAUD, 1975 ; GARREAU, 1976) (') :

- une intervention profonde (labour ou chisel) effectuée en été le plus tôt possible pour qu'il y ait fragmentation par l'outil, puis fissuration sous l'action des alternances humectation-dessication,

- préparation des lits de semence, rigolage $\left({ }^{2}\right)$ et désherbage avant la période d'excès d'eau, y compris pour les cultures de printemps, ceci pour minimiser le nombre de passages sur profil humide.

A l'échelle de l'exploitation, la mise en cuvre de ces techniques conduit à une concentration des travaux en été-début automne. Il en résulte des reports de travaux inévitables par rapport aux recommandations.

L'enregistrement des techniques culturales pratiquées dans les exploitations a confirmé l'existence de tels écarts (CAPILlon \& Pellerin, 1984). Dès lors, pour raisonner la conduite du travail du sol dans l'exploitation, il importe de connaître non pas tant les conditions optimales d'intervention que le moment à partir duquel l'état obtenu devient nettement défavorable.

Dans cet esprit le présent travail étudie l'effet de la date de labour sur l'état structural de l'horizon labouré, non repris par les façons superficielles ultérieures. Dans la suite du texte et pour alléger la présentation on parlera de «l'horizon labouré » pour désigner l'horizon ainsi défini.

A priori, et pour un état initial donné, la date de labour conditionne l'état structural créé dans l'horizon labouré à travers

- l'état hydrique au moment du labour, dont dépend le comportement mécanique du matériau sous l'action de l'outil donc l'état sitôt après labour,

- les possibilités d'évolution ultérieures de cet état sous l'action du climat, notamment par fissuration.

Un $1^{\text {er }}$ suivi portant sur 19 parcelles de maïs et réalisé en situation d'enquête a de fait révélé une différence importante entre parcelles labourées avant et après réhumectation du profil (PELLERIN, 1984). Par contre il n'est pas apparu d'effet date entre différents labours faits sur profils secs.

Pour interpréter ces résultats il importe de dissocier dans l'effet date ce qui est dû à l'état hydrique au moment du labour de ce qui est dû à l'évolution après labour sous l'action du climat.

Pour cela et compte tenu des caractéristiques climatiques de la région, l'état structural créé dans l'horizon labouré et son évolution ont été comparés pour les 3 situations suivantes :

( $\left.{ }^{1}\right)$ Plusieurs types de sols, se distinguant notamment par la stabilité structurale du matériau, ont été identifiés en Marais. On distingue :

— des sols argileux humifères, très stables,

- des sols argileux stables,

- des sols argileux sodiques dispersants,

- quelques sols tourbeux.

Les techniques culturales préconisées ici sont adaptées aux sols argileux stables et très stables. Les sols sodiques dispersants sont peu mis en culture.

(2) A l'échelle parcellaire l'évacuation des excès d'eau est assurée par un profilage en ados, de 18 ou $27 \mathrm{~m}$, et l'ouverture de rigoles. 
- deux labours, notés L1 et L2, faits sur profil sec à même teneur en eau mais à des dates différentes, donc soumis après labour à une durée d'évolution sous l'action du climat différente,

- un labour, noté $\mathrm{L} 3$, fait après réhumectation du profil à une teneur en eau proche de la capacité au champ.

Les situations intermédiaires et l'effet de la date de labour sur l'horizon repris par les façons superficielles ne sont pas abordés dans cet article.

\section{MATÉRIEL ET MÉTHODES}

\section{A. Le dispositif}

Les dates de labour des parcelles suivies sont indiquées dans le tableau 1. L'année climatique $82 / 83$ a été caractérisée par un été sec, puis un brutal retour des pluies le 23 septembre (fig. 1). On a ainsi :

- Trois traitements A1/A2/A3 correspondant aux 3 situations $\mathrm{L} 1 / \mathrm{L} 2 / \mathrm{L} 3$. Au moment du labour les teneurs en eau pondérales moyennes dans les 30 premiers $\mathrm{cm}$ étaient de 0,24 et $0,22 \mathrm{~g} / \mathrm{g}$ en $\mathrm{Al}$ et $\mathrm{A} 2$, de $0,37 \mathrm{~g} / \mathrm{g}$ en $\mathrm{A} 3$, soit une teneur en eau proche de la capacité au champ (annexe 1).

- Deux autres couples de traitements B1/B3 et $\mathrm{C} 1 / \mathrm{C} 2$ correspondant respectivement aux situations $\mathrm{L} 1 / \mathrm{L} 3$ et $\mathrm{L} 1 / \mathrm{L} 2$.

Pour chaque couple (ou triplet) de traitements, l'itinéraire technique après labour a été aussi identique que possible (tabl. 1).

Les traitements destinés à être comparés ont été placés sur des parcelles proches quant à leur histoire culturale et leur situation hydraulique. Les parcelles se situent dans des zones isolées hydrauliquement où l'eau collectée dans les rigoles puis les fossés est évacuée par pompage.
L'analyse de terre ne révèle pas de différence importante entre les parcelles destinées à être comparées (tabl. 2). Les sols sont argileux, dessalés et très peu sodiques.

Les données analytiques permettent de pronostiquer une stabilité structurale élevée et peu différente entre parcelles devant être comparées, résultat confirmé par le test de percolation (indice log $10 \mathrm{KI}$, HENIN et al., 1969 ; HENIN, 1976).

\section{B. Le choix des observations et des contrôles}

L'objectif est :

- de classer les traitements quant à l'état structural obtenu dans l'horizon labouré quand est implantée la culture ;

- de rechercher l'origine de ce classement.

Le calendrier des observations et mesures réalisées est indiqué en figure 2. L'état structural de l'horizon labouré a été décrit après semis. Des observations sur la culture, cartographie d'enracinement et mesures de croissance, ont été effectuées pour corroborer ou infirmer le classement établi. Le maïs a été choisi pour sa sensibilité à l'état du profil.

Pour discuter de l'origine des états obtenus après semis, plusieurs observations successives du profil cultural ont été réalisées entre labour et semis dans les traitements $\mathrm{A} 1, \mathrm{~A} 2$ et $\mathrm{A} 3$.

Après repérage de l'horizon labouré non concerné par les reprises superficielles, l'état structural de celuici a été décrit par cartographie des unités morphologiques du profil selon une méthode proposée par MANICHON (1982a-b). Les zones identifiées sont caractérisées par le mode d'assemblage des éléments structuraux constitutifs et leur état interne (tabl. 3). En termes de porosité les différents états s'ordonnent comme suit :

$-\Gamma>\Delta$ (un état intermédiaire noté $\Gamma \Delta$ a été utilisé. Il se caractérise par des agrégats constitutifs plus ou moins fondus entre eux),

TABLEAU 1

Itinéraire technique des parcelles suivies. Cultivation history of each plot.

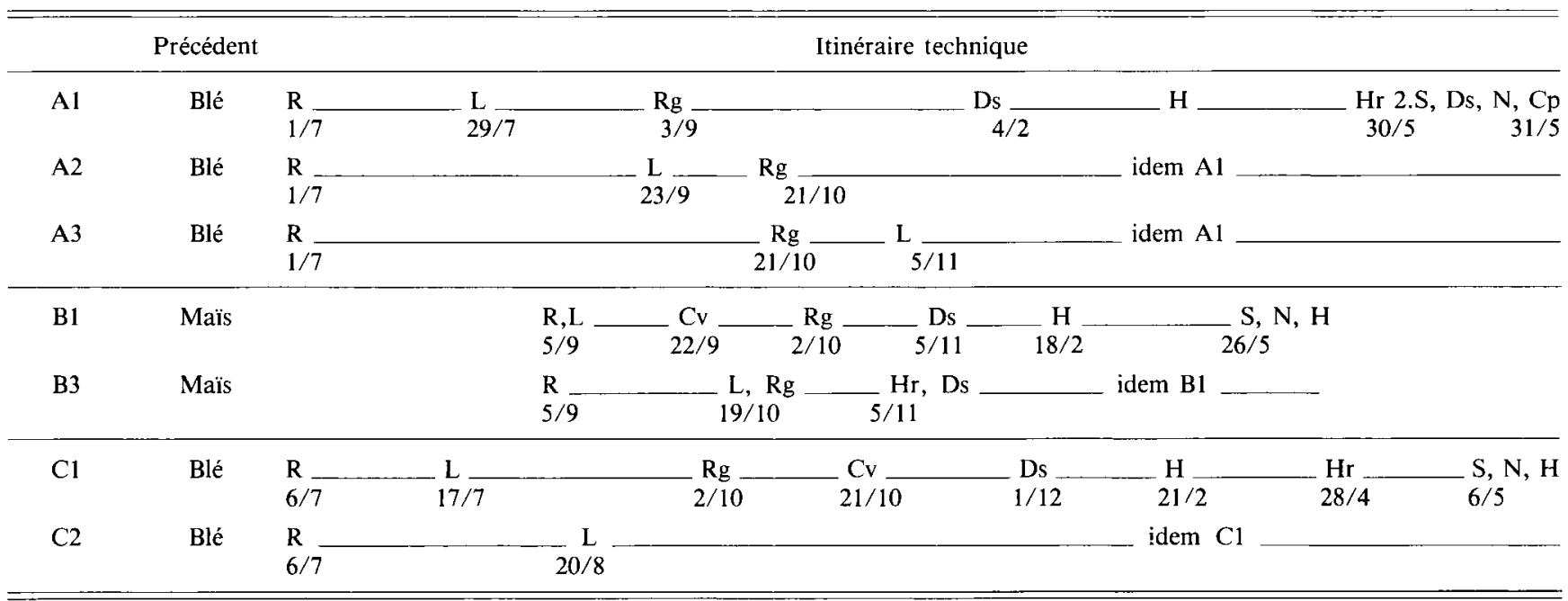

R : Récolte du précédent ; Harvest. L : Labour ; Ploughing. Rg : Rigolage ; Furrow drain. Ds : Désherbage ; Weed control. H : Hersage ; Harrowing. $\mathrm{Hr}$ : Passage herse rotative ; rotary harrow. S : Semis ; Sowing. N : Apport d'engrais, Fertilization. Cp : Passage cultipacker ; Cultipacker. $\mathrm{Cv}$ : Passage cultivateur ; Tiller. 
TABLEAU 2

Analyse de sol et test de stabilité structurale. Horizon $0-30 \mathrm{~cm}$.

Soil analysis and soil structure stability. Horizon 0-30 cm.

\begin{tabular}{|c|c|c|c|c|c|}
\hline Parcelles & Al & $\mathrm{A} 2 / \mathrm{A} 3(1)$ & B1 & B3 & $\mathrm{C} 1 / \mathrm{C} 2(1)$ \\
\hline \multicolumn{6}{|l|}{ Granulométrie } \\
\hline - sables grossiers & 0,9 & 0,8 & 1,3 & 0,8 & 0,6 \\
\hline — sables fins & 0,7 & 1,1 & 2,7 & 0,8 & 0,7 \\
\hline - sables très fins & 2,3 & 3,7 & 10,2 & 6,4 & 3,2 \\
\hline - limons & 31,7 & 32,6 & 30,2 & 36,0 & 30,2 \\
\hline — argiles & 60,9 & 57,7 & 52,6 & 52,9 & 61,3 \\
\hline $\mathrm{PH}$ & 8,1 & 8,0 & 8,2 & 8,2 & 8,2 \\
\hline $\mathrm{CaCO}_{3}$ total $(\mathrm{g} / 100 \mathrm{~g})$ & 2 & 3 & 7 & 7 & 9 \\
\hline Matière organique & 4,3 & 3,3 & 3,7 & 3,3 & 3,1 \\
\hline CEC (meq/100 g) & 31,88 & 32,78 & 28,03 & 29,73 & 33,00 \\
\hline \multicolumn{6}{|l|}{ Cations échangeables } \\
\hline$-\mathrm{Ca}^{++}$ & 21,37 & 25,89 & 21,29 & 28,35 & 24,21 \\
\hline$-\mathrm{Mg}^{++}$ & 8,35 & 5,07 & 4,86 & 4,40 & 6,80 \\
\hline$-\mathrm{Na}^{+}$ & 0,57 & 0,31 & 0,36 & 0,29 & 0,57 \\
\hline$-\mathrm{K}^{+}$ & 1,59 & 1,51 & 1,52 & 1,19 & 1,42 \\
\hline \multicolumn{6}{|l|}{ Cations solubles } \\
\hline$-\mathrm{Ca}^{++}$ & 1,94 & 2,45 & 2,59 & 2,59 & 2,82 \\
\hline$-\mathrm{Mg}^{++}$ & 0,83 & 0,54 & 0,57 & 0,48 & 0,83 \\
\hline$-\mathrm{Na}^{+}$ & 0,07 & 0,00 & 0,04 & 0,01 & 0,07 \\
\hline$-\mathbf{K}^{+}$ & 0,18 & 0,15 & 0,16 & 0,08 & 0,20 \\
\hline $\mathrm{Nae} / \mathrm{T}(\%)(2)$ & 1,8 & 1,0 & 1,3 & 1,0 & 1,7 \\
\hline $\log .10 \mathrm{~K} 1$ & 1,93 & 1,95 & 1,56 & 1,46 & 1,46 \\
\hline
\end{tabular}

(1) Ces 2 traitements ont été placés sur une même parcelle.

(2) Taux de sodium échangeable rapporté à la capacité d'échange.

(1) Those two treatments were placed on the same plot.

(2) Exchangeable sodium, related to total exchange capacity.

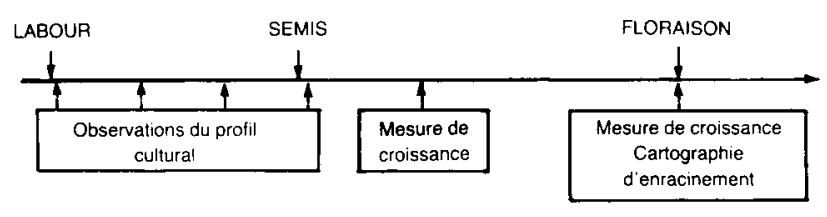

Figure 2

Calendrier des observations et mesures réalisées.

Measurements and observations made.

TABLEAU 3

Modes d'assemblage des éléments structuraux et types d'état interne (d'après MANICHON, 1982a-b).

Notations used to describe profiles: internal states of clods and way they are brought together (after MANICHON, 1982a-b).

\begin{tabular}{lc}
\hline \multicolumn{1}{c}{ Modes d'assemblage } & Notation \\
\hline - Fragmentaire, mottes individualisées & $\mathrm{F}$ \\
- Mottes soudées, à contours facilement discernables & $\mathrm{SF}$ \\
- Mottes soudées, à contours difficilement discernables & $\mathrm{SD}$ \\
- Massif & $\mathrm{M}$ \\
- Variante par rapport aux états F, SF et SD indiquant & $\mathrm{FV}$, \\
la présence de cavités importantes (plus de $5 \mathrm{~mm}$ ) & $\mathrm{SFV}$, \\
entre mottes & $\mathrm{SDV}$ \\
\hline
\end{tabular}

Etats internes et Identification

- On distingue les agrégats constitutifs de l'élément face de rupture rugueuse

- Il y a eu coalescence des agrégats constitutifs - face de rupture peu rugueuse, d'aspect continu, de forme conchoïdale

$-\Delta$, avec apparition de fissures de retrait
- pour un même état interne, $\mathrm{F}>\mathrm{SF}>\mathrm{SD}>\mathrm{M}$.

Pour contrôler la validité des observations visuelles d'état interne, celles-ci ont été réalisées sur une série de mottes de 4 à $6 \mathrm{~cm}$ de diamètre dont on a mesuré le volume massique $\left(1 / \gamma \mathrm{d}\right.$ en $\left.\mathrm{cm}^{3} / \mathrm{g}\right)$ et la teneur en eau pondérale ( $W$ en $\mathrm{g} / \mathrm{g}$ ou $\mathrm{cm}^{3} / \mathrm{g}$ ).

Les résultats des observations d'état interne, complétés par une note visuelle de porosité de 0 à 4 ont été reportés sur les points figurant les échantillons dans le système d'axes ( $\mathrm{W}, 1 / \gamma \mathrm{d})$ (fig. 3).

Les points se disposent en gros selon une parallèle à la première bissectrice. Dans la gamme d'humidité étudiée le retrait des mottes est assimilable à un retrait normal (HAINES, 1923).

Pour une même teneur en eau il existe une gamme de volumes massiques mesurés. Cette gamme ne peut a priori correspondre qu'à des variations du volume poral structural intra-motte. Or on constate, à quelques exceptions près, que les échantillons s'ordonnent en fonction de leur état interne. Ce résultat valide les observations visuelles réalisées pour classer les échantillons en termes de porosité structurale.

L'écart à la droite de saturation, calculée pour une densité de solide $\gamma \mathrm{s}=2,70 \mathrm{~g} / \mathrm{cm}^{3}$, mesure le volume poral rempli d'air. Pour chaque échantillon l'indice d'air Vair $\left(\mathrm{cm}^{3} / \mathrm{g}\right)$ a pu être calculé :

$$
\text { Vair }=1 / \gamma d-1 / \gamma s-W .
$$

Dans la gamme de teneur en eau étudiée on constate que le volume poral rempli d'air est important en $\Gamma$, faible et relativement constant en $\Gamma \Delta$ et proche de 


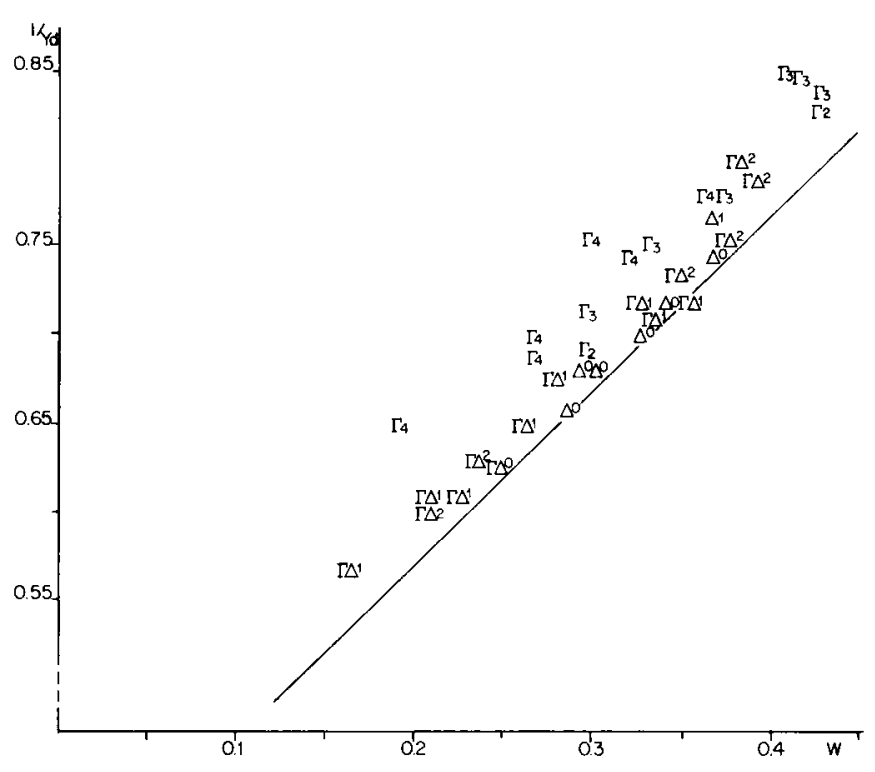

Figure 3

Volumes massiques de mottes $(1 / ; d)$, caractérisées par leur état interne et une note visuelle de porosité (de 0 à 4), en fonction de leur teneur en eau pondérale $(W)$.

Mass/volume ratio of clods $(1 / \gamma d)$, described by their internal state and porosity, related to their water content $(W)$.

zéro en $\Delta$. Ces mottes $\Gamma \Delta$ et $\Delta$ restent proches de la saturation même aux humidités les plus faibles, ce qui indiquerait que le point d'entrée d'air dans la porosité texturale n'est pas atteint. Ce constat est cohérent avec des résultats obtenus par ailleurs pour un sol de constitution assez proche et dont la teneur en eau mesurée au point d'entrée d'air est inférieure à $0,20 \mathrm{~g} / \mathrm{g}$ (CARDON-DuBOIS, 1985). Ce résultat sera repris en discussion.

L'enracinement a été cartographié par observation d'une face de profil en utilisant une grille de mailles carrées de $2,5 \mathrm{~cm}$ dans lesquelles on note l'abondance de racines (notes croissantes de 0 à 5) et l'état structural (TARDIEU, 1984). On caractérise ainsi la répartition des racines que l'on confronte aux hétérogénéités porales du profil. Pour pouvoir comparer les traitements à cet égard il était nécessaire d'effectuer l'observation à même densité de peuplement dans l'environnement immédiat du profil. L'emplacement de celui-ci a été choisi en ce sens dans la mesure du possible. Le nombre de pieds et leur état de croissance à proximité immédiate de la face d'observation ont fait l'objet d'un contrôle. Enfin l'observation a été réalisée au voisinage de la floraison femelle, période à laquelle le système racinaire atteint son état de croissance maximum (MENGEL \& BARBER, 1974).

Les mesures de croissance ont été effectuées vers le 10 juillet, après une période de fort déficit hydrique (fig. 1), puis à une date proche de la floraison femelle. On a utilisé un indicateur non destructif de l'état de croissance. Sur des placettes tirées au hasard correspondant à un rang de maïs sur $3 \mathrm{~m}$ de long on a mesuré :

- les espacements entre pieds,

- le stade, la hauteur (h) et le diamètre $(\varnothing)$ de chacun des pieds. Pour un stade donné et jusqu'à floraison le critère $h \varnothing^{2}$ est un bon indicateur de

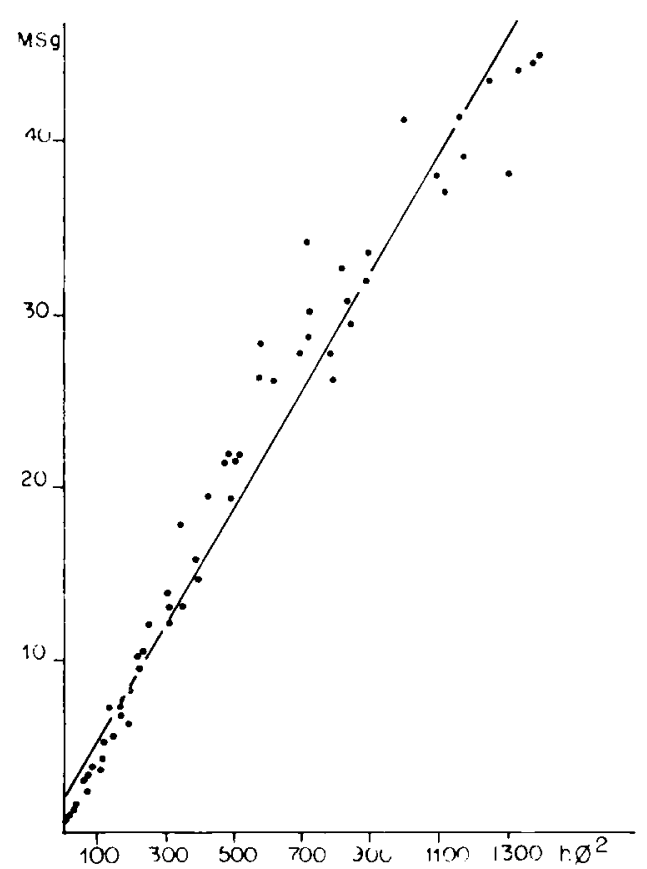

Figure 4

Matière sèche par pied (en $\mathrm{g}$ ), en fonction du critère $H \varnothing^{2}\left(e n \mathrm{~cm}^{3}\right)$. Parcelles A1, A2, A3-Prélèvement le 10/07/83

$$
M S=1,773+0,035^{*} H \varnothing^{2} \quad r^{2}=0,96
$$

Dry matter per plant $(\mathrm{g})$, related to calculated $H \varnothing^{2}\left(\mathrm{~cm}^{3}\right)$ - Plots $A 1$, A2, A3 - Plants sampled on 10/07/83

$$
M S=1.773+0.035 * H \varnothing^{2} \quad r^{2}=0,96
$$

la matière sèche par pied (TARDIEU F., comm. pers., 1983). Pour la $1^{\text {re }}$ série de mesure, la plus importante pour notre dispositif, l'existence de cette liaison a été vérifiée (fig. 4).

\section{RÉSULTATS}

\section{A. Le classement des états structuraux observés après semis et résultats sur la culture}

Le classement des états structuraux observés après semis dans l'horizon labouré non repris est indiqué dans le tableau 4.

TABLEAU 4

Importance (en \% de la surface du profil) que représentent les différents modes d'assemblage et états internes. Profil observé après semis.

Relative importance of different typical assemblages of clods and internal states observed in the profiles. Profile observed after sowing.

\begin{tabular}{lccccccc} 
& A1 & A2 & A3 & B1 & B3 & C1 & C2 \\
\hline SF $\Gamma$ & 76 & 91 & 23 & 69 & 40 & 84 & 82 \\
SD $\Gamma$ & - & - & - & - & 23 & - & - \\
SD $\Gamma \Delta$ & 11 & 2 & 62 & 7 & 4 & 8 & 10 \\
M $\Gamma$ & - & - & - & - & - & 8 & - \\
M $\Gamma \Delta$ & 14 & 7 & 14 & 18 & 17 & - & 8 \\
M $\Delta$ & - & - & 1 & 5 & 17 & - & - \\
Total & 100 & 100 & 100 & 100 & 100 & 100 & 100 \\
\hline \hline
\end{tabular}


Il n'apparaît pas de différence importante entre les labours faits sur sol sec à des dates différentes (couples $\mathrm{A} 1 / \mathrm{A} 2$ et $\mathrm{C} 1 / \mathrm{C} 2)$. L'état structural dominant est noté SF, d'état interne $\Gamma$.

Par contre, dans le cas des traitements A3 et B3, des zones notées $\mathrm{M}$ ou $\mathrm{SD}$, d'état interne dégradé $(\Gamma \Delta$, voire $\Delta)$, apparaissent dans le profil.

Les résultats des cartographies d'enracinement pour les parcelles $\mathrm{B} 1, \mathrm{~B} 3, \mathrm{C} 1$ et $\mathrm{C} 2$ sont résumés dans le tableau 5. Les résultats concernant les parcelles $\mathrm{Al}$, $\mathrm{A} 2$ et $\mathrm{A} 3$ sont difficilement utilisables du fait des différences de densité de peuplement entre parcelles (tabl. 6). Il n'a pas été possible de réaliser l'observation sans qu'il y ait en $\mathrm{A} 2$ et $\mathrm{A} 3$ des pieds manquant à proximité de la face cartographiée. Cela a par contre été possible en $\mathrm{B} 1, \mathrm{~B} 3, \mathrm{C} 1$ et $\mathrm{C} 2$. On vérifie que le nombre de pieds et leur état de croissance moyen dans l'environnement immédiat du profil ne sont pas très différents entre traitements (tabl. 7).

On observe dans le cas de la parcelle B3 des zones -non ou peu colonisées qui correspondent aux zones d'état structural dégradé (fig. 5). A noter qu'à cette date d'observation il y a eu soudure de tous les éléments structuraux du profil et on distingue seulement des différences d'état interne. La fréquence des cases

TABLEAU 5

Résultats des cartographies d'enracinement Parcelles Bl, B3, $\mathrm{Cl}, \mathrm{C} 2$.

Results of roots maps. Plots B1, B3, C1, C2.

$\begin{array}{llll}\mathrm{B} 1 & \mathrm{~B} 3 & \mathrm{C} 1 \quad \mathrm{C} 2\end{array}$

Pourcentage de case avec absence de racine

- Horizon 10-30

- Horizon 20-30

$27,9 \quad \underline{31,9} \quad 27,1 \quad 28,8$

$18,7 \quad \underline{32,1} \quad 27,7 \quad 23,0$

Pourcentage des cases sans racine $\begin{array}{lllll}\text { contiguës d'une autre case sans racine } \quad 90,3 & \underline{95,4} & 89,2 & 92,4\end{array}$

TABLEAU 6

Densité de peuplement dans les parcelles suivies. Plant density in plots studied.

\begin{tabular}{cccccc}
\hline \hline Parcelle & $\mathrm{r}$ & $\mathrm{e}$ & $\mathrm{dt}$ & $\mathrm{p}$ & $\mathrm{dr}$ \\
\hline $\mathrm{A} 1$ & 0,80 & 0,145 & 87000 & 0,73 & 65000 \\
\hline $\mathrm{A} 2$ & 0,80 & 0,145 & 87000 & 0,59 & 52000 \\
\hline $\mathrm{A} 3$ & 0,80 & 0,145 & 87000 & 0,47 & 42000 \\
\hline B1 & 0,75 & 0,150 & 88000 & 0,87 & 77000 \\
\hline $\mathrm{B} 3$ & 0,75 & 0,150 & 88000 & 0,91 & 82000 \\
\hline $\mathrm{C} 1$ & 0,75 & 0,135 & 100000 & 0,72 & 76000 \\
\hline $\mathrm{C} 2$ & 0,75 & 0,135 & 100000 & 0,88 & 93000 \\
\hline \hline
\end{tabular}

$\mathrm{r}=$ écartement entre rangs (en $\mathrm{m}$ ) - Spacing between rows ( $\mathrm{m}$ ).

$\mathrm{e}=$ écartement de réglage entre pieds (en $\mathrm{m}$ ) - Theoretical spacing between plants $(\mathrm{m}$.)

$\mathrm{dt}=$ densité théorique de semis (grains par ha) - Theoretical density of sowing (kernels per ha).

$\mathrm{p}=$ taux de présence $=$ nombre de pieds/nombre de graines Number of plants relative to number of kernels.

$\mathrm{dr}=$ densité réelle (pieds par ha) - Real density (plants per ha).

\section{TABLEAU 7}

Nombre de pieds présents (n) sur les 14 emplacements les plus proches de la face d'observation 7 emplacements situés de part et d'autre de la face observée sur les 2 rangs correspondant au profil) et état de croissance moyen de ces pieds ( $h \varnothing^{2}$ moyen en $\mathrm{cm}^{3}$ ).

Number of plants (n) at the 14 seed locations close to the observed profile face $(7$ on either side of the face, in the two rows corresponding to the profile) and mean growth of these plants $\left(h \varnothing^{2}\right)$.

\begin{tabular}{ccc}
\hline Parcelle & $\mathrm{n}$ & $\mathrm{h} \varnothing^{2}$ moyen \\
\hline B1 & 14 & 1266 \\
\hline $\mathrm{B} 3$ & 14 & 1128 \\
\hline $\mathrm{C} 1$ & 14 & 1521 \\
\hline $\mathrm{C} 2$ & 14 & 1384 \\
\hline
\end{tabular}
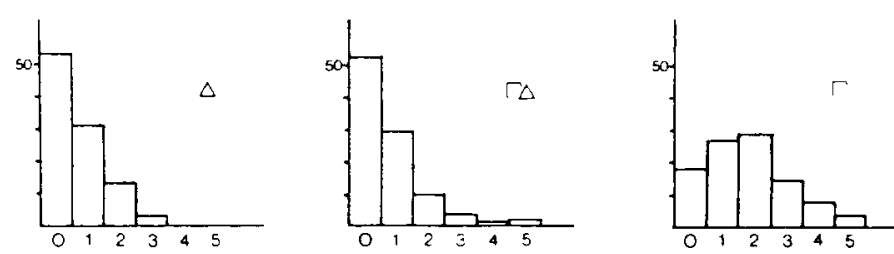

Figure 5

Distribution des notes d'enracinement, par type d'état interne. Cas du traitement B3.

Distribution of rooting scores, for each internal state of clods. Case of treatment $B 3$.

avec absence de racine est plus élevée en B3 qu'en B1 et celles-ci sont plus souvent groupées (tabl. 5). En $\mathrm{B} 1$, ces cases non colonisées sont surtout situées dans l'horizon 10-20 cm sous l'interrang, alors qu'en B3 leur fréquence reste élevée dans l'horizon $20-30 \mathrm{~cm}$. Il a pu en résulter, par effet "d'ombrage " (TARDIEU, 1984), une moindre colonisation du sous-sol.

Les résultats de la $1^{\text {re }}$ mesure de croissance sont consignés dans la figure 6 . A cette date, on observe encore peu d'effet de la densité de peuplement sur la croissance moyenne par pied (fig. 6). On peut donc comparer les traitements quant à l'état de croissance moyen par pied sans tenir compte des variations de densité. On observe

- peu de différence de croissance entre les traitements $\mathrm{A} 1$ et $\mathrm{A} 2$, d'une part, $\mathrm{C} 1$ et $\mathrm{C} 2$, d'autre part. les moyennes calculées sont non significativement différentes dans les 2 cas (tabl. 8),

\section{TABLEAU 8}

Première mesure de croissance : comparaison des traitements. First growth measurement : comparison of treatments.

\begin{tabular}{ccccc}
\hline $\mathrm{A} 1 / \mathrm{A} 2$ & $\mathrm{~A} 1 / \mathrm{A} 3$ & $\mathrm{~A} 2 / \mathrm{A} 3$ & $\mathrm{~B} 1 / \mathrm{B} 3$ & $\mathrm{C1} / \mathrm{C} 2$ \\
\hline $\mathrm{NS}$ & $\mathrm{S}$ & $\mathrm{S}$ & $\mathrm{S}$ & $\mathrm{NS}$ \\
\hline
\end{tabular}

A1/A2/A3 : Test T corrigé ; niveau global de risque ; $\alpha=0,10$; BONFERRONI test; global level or risk, $=0.10$.

B1/B3 : Test T; $\alpha=0,10$; STUdent test ; $\alpha=0.10$.

$\mathrm{C} 1 / \mathrm{C} 2$ : Test $\mathrm{T} ; \alpha=0,10$; STUDENT test $; \alpha=0.10$.

$\mathrm{S}$ : différence significative $(\alpha=0,1)$; NS : différence non significative. 


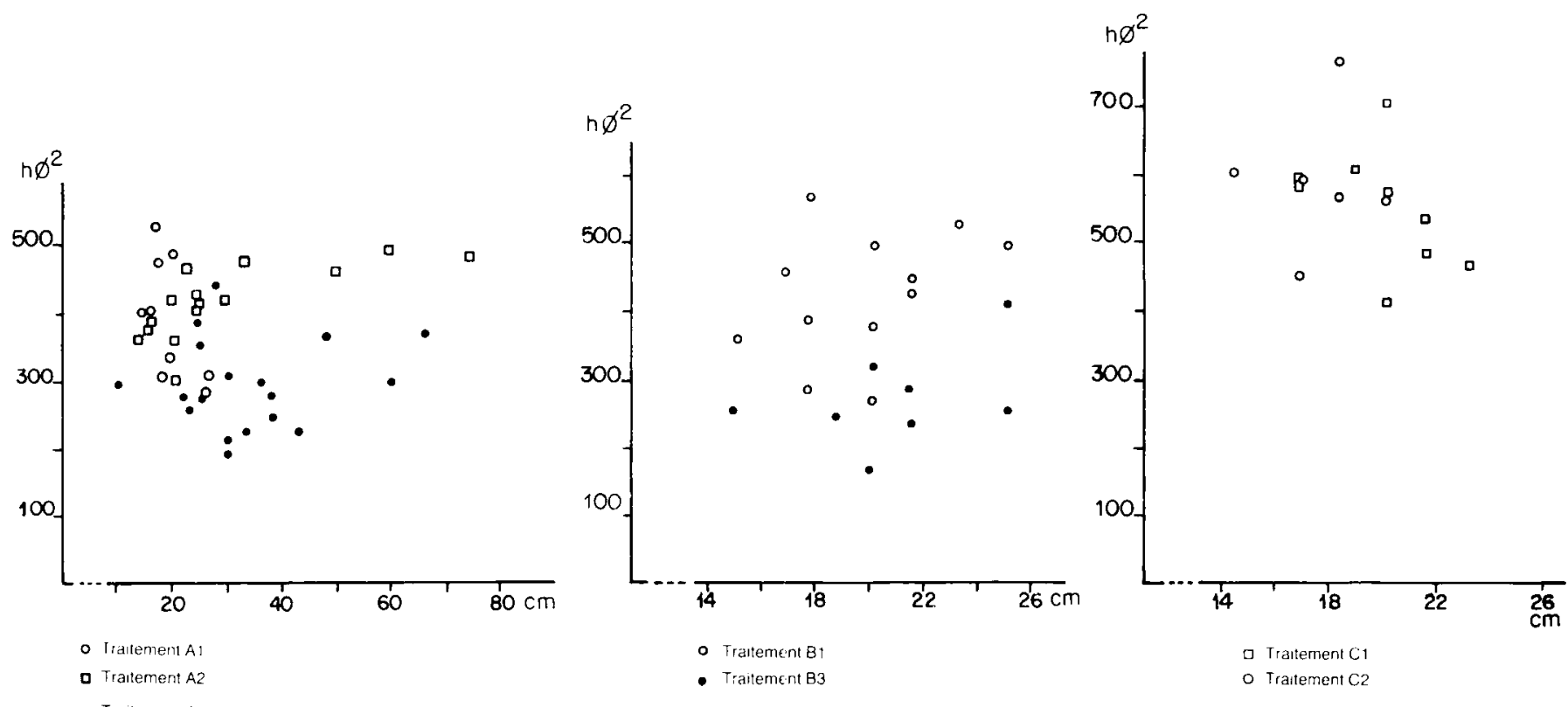

Figure 6

Etat de croissance moyen par pied, pour chaque placette, en fonction de l'espacement moyen entre pieds. Première mesure.

Mean growth per plant, related to mean spacing between plants. First measurement.
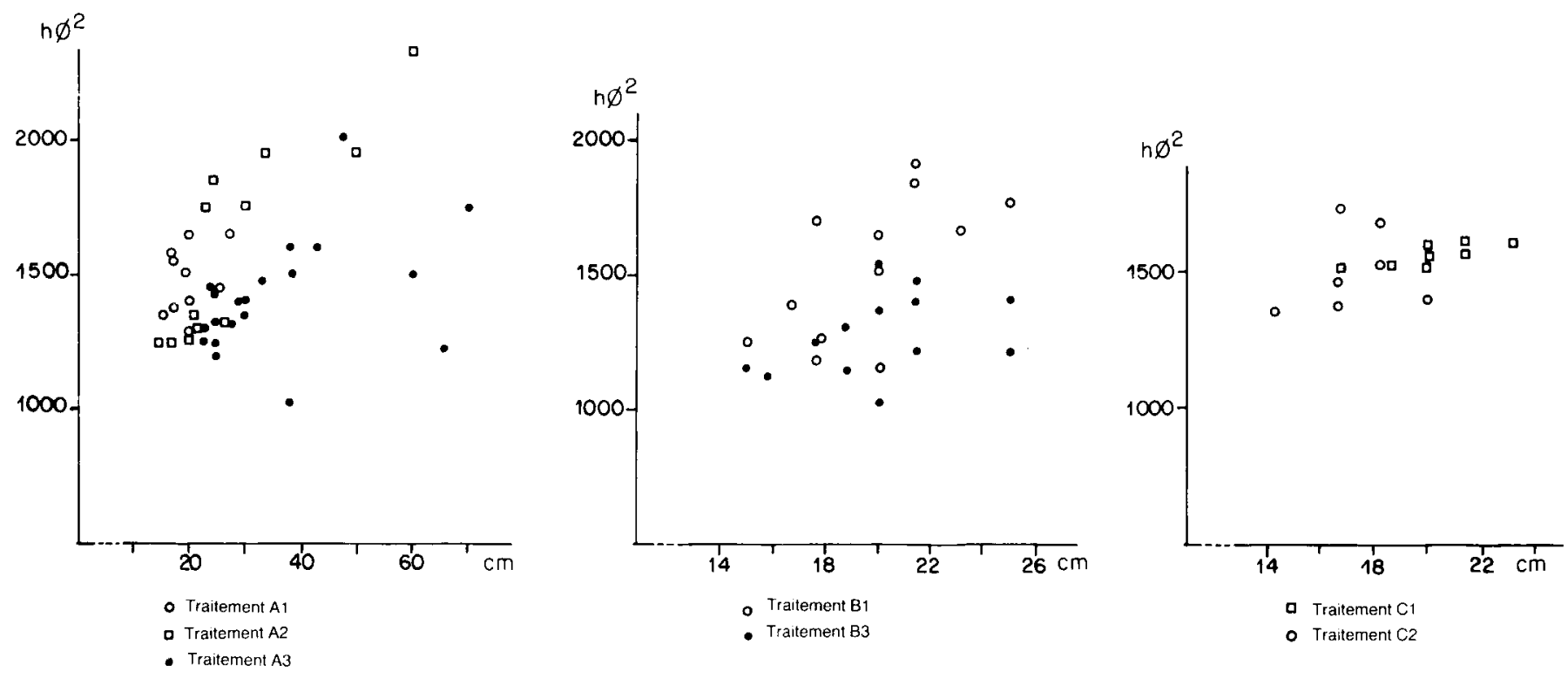

Figure 7

Etat de croissance moyen par pied, pour chaque placette, en fonction de l'espacement moyen entre pieds. Deuxième mesure.

- un fort déficit de croissance pour les traitements A3 et B3, confirmé par les mesures de croissance effectuées à floraison (fig. 7).

Ces résultats sont convergents. Pour les couples de parcelles où cela a été testé on a, vis-à-vis de la qualité de l'état structural créé dans l'horizon labouré, $\mathrm{L} 1=\mathrm{L} 2>\mathrm{L} 3$.

\section{B. L'origine de ce classement : les observations inter-} médiaires (tabl. 9)

Après labour en A1 et A2 l'état structural dominant est fragmentaire, constitué de mottes inférieures à
Mean growth per plant, related to mean spacing between plants. Second measurement.

$15 \mathrm{~cm}$ de diamètre, d'état interne $\Gamma$, ménageant entre elles des vides importants. On observe également quelques gros blocs, notés $\mathrm{M} \Gamma$.

Avant le début des pluies le 23 septembre cet état reste inchangé, quelle que soit la date d'observation (cas de A1). L'humidité au sein de l'horizon labouré varie peu. Ainsi le 14 septembre, après une période de fort déficit hydrique (fig. 1), la teneur en eau moyenne mesurée dans l'horizon labouré $(10-30 \mathrm{~cm})$ était de $0,23 \mathrm{~g} / \mathrm{g}$.

Après réhumectation du profil on constate un rapprochement des éléments structuraux (passage d'un état $\mathrm{F}+$ vides à un état $\mathrm{F}$ puis à.un état $\mathrm{SF}$ ) sans modification d'état interne. 
TABLEAU 9

Modes d'assemblage des éléments structuraux et types d'état interne observés dans les profils à différentes dates. Traitements A1, A2 et A3.

Typical assemblages and internal states of clods observed in the profiles at various dates.

Treatments $A 1, A 2$ and $A 3$.

\begin{tabular}{|c|c|c|c|c|c|c|c|c|}
\hline Traitement & Dates & $\mathrm{M \Gamma}$ & МГ $\Delta$ & FVI & $\mathbf{F} \Gamma$ & $\mathrm{SF} \Gamma$ & SDr & SDI $\Delta$ \\
\hline \multirow{5}{*}{ Al } & $18 / 08$ & $*(1)$ & & $* * * *$ & & & & \\
\hline & $14 / 09$ & $*(1)$ & & $* * * *$ & & & & \\
\hline & $12 / 10$ & ${ }^{*}(2)$ & & & $* * * *$ & & & \\
\hline & $21 / 10$ & $*(2)$ & & & $* * * *$ & & & \\
\hline & $21 / 02$ & $*$ & $*$ & & & $* * *$ & $*(3)$ & \\
\hline \multirow{4}{*}{ A2 } & $24 / 09$ & $*(1)$ & & $* * * *$ & & & & \\
\hline & $12 / 10$ & ${ }^{*}(1)$ & & $* * * *$ & & & & \\
\hline & $21 / 10$ & $*$ & & & $* * * *$ & & & \\
\hline & $21 / 02$ & $*$ & & & & $* * * *$ & & \\
\hline \multirow{2}{*}{ A3 } & $05 / 11$ & & & & $* *$ & * & ** & \\
\hline & $22 / 02$ & & & & & $* *$ & $*$ & $* *$ \\
\hline
\end{tabular}

****: Plus de $75 \%$ du profil - More than $75 \%$ of the profile.

*** : 50 à $75 \%$ - 50 to $75 \%$

**: 25 à $50 \%-25$ to $50 \%$.

*: Moins de $25 \%$ - Less than $25 \%$

(1) Gros blocs (> a $15 \mathrm{~cm}$ ) - Large blocks (more than $15 \mathrm{~cm}$ ).

(2) Mêmes gros blocs, fissurés sur $5 \mathrm{~cm}$ en surface. Same large blocks, cracked on upper $5 \mathrm{~cm}$.

(3) Tendance à un état $\Gamma \Delta$ par endroit. Tendency to the internal state noted $\Gamma \Delta$

En A3 les bandes de labour sont moulées, moins fragmentaires que dans le cas précédent. L'état structural dominant est noté SD, d'état interne $\Gamma$, avec quelques zones SF ou $\mathrm{F}$.

Après l'hiver on retrouve :

- des zones notées SF, d'état interne $\Gamma$,

- des zones notées SD, avec un état interne dégradé $\Gamma \Delta$.

Ces zones d'état interne dégradé ont été observées même en dehors des passages de tracteur et ne peuvent donc pas correspondre aux seules actions de tassement par les roues. Tout se passe comme si le mode d'assemblage différent obtenu après labour entre A1 et A2, d'une part, A3, d'autre part, avait induit des évolutions différentes d'état interne.

\section{DISCUSSION}

Le classement obtenu ( $\mathrm{L} 1=\mathrm{L} 2>\mathrm{L} 3)$ ne concerne que quelques situations extrêmes et est contingent du climat de l'année de suivi. En cela il n'est pas extrapolable. Cependant,

- l'année 1982/83 a été très proche des conditions climatiques moyennes (fig. 1),

- le classement obtenu est convergent avec les résultats de l'année précédente (PELLERIN, 1984).

D'après nos observations, la fissuration sous l'action des alternances humectation-dessiccation, importante en surface, concerne peu l'horizon sousjacent $(10-30 \mathrm{~cm})$.

Dans les sols de Marais, la fissuration visible apparaît surtout lors de la réhumeetation. Des travaux récents effectués en laboratoire sur des échantillons initialement malaxés et saturés d'un matériau de cons- titution assez proche montrent que la fissuration à la réhumectation n'intervient que s'il y a eu dessiccation préalable en dessous d'un seuil de teneur en eau, qui semble correspondre au point d'entrée d'air dans la porosité texturale, et si la réhumectation se fait au contact d'eau libre ou soumise à une succion faible (CARDON - DubOIS, 1985 ; P. STENGEL, comm. pers., 1986).

Sous réserve que ces résultats puissent être extrapolés au champ et à notre matériau, on peut penser que le mulch créé par les mottes ayant été fissurées en surface protège l'horizon sous-jacent d'une dessiccation suffisamment forte pour qu'il y ait fissuration. De fait, la teneur en eau mesurée dans l'horizon $10-30 \mathrm{~cm}$ en $\mathrm{A} 1$, après une période de fort déficit hydrique (le 14 septembre) était encore de $0,23 \mathrm{~g} / \mathrm{g}$, humidité à laquelle le point d'entrée d'air dans la porosité texturale n'apparaît pas atteint (fig. 3).

L'avantage des labours précoces vis-à-vis de l'état structural de l'horizon labouré non repris ne serait donc pas lié aux possibilités de fissuration ultérieures sous l'action du climat.

Par contre, tout se passe comme si l'état fragmentaire obtenu après labour en $\mathrm{A} 1$ et $\mathrm{A} 2$ avait autorisé, plus qu'en $\mathrm{A} 3$, le maintien après hiver d'un état interne $\Gamma$.

Compte tenu de la richesse du matériau en argiles gonflantes on peut avancer l'hypothèse suivante : soit une motte $\Gamma$ soumise à gonflement. On peut imaginer 2 cas (fig. 8) :

- ou bien son volume apparent n'est pas limité (cas 1), auquel cas le gonflement peut à priori se faire

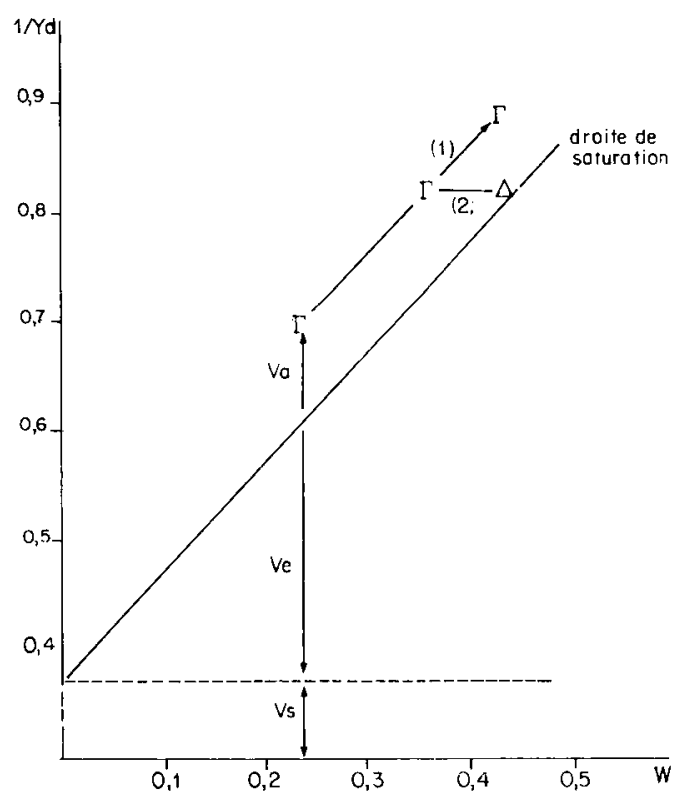

Figure 8

Evolution supposée d'une motte d'état initial $\Gamma$ (volume poral initial rempli d'air non nul), soumise à gonflement en volume contraint (cas 2) ou non contraint (cas 1).

Supposed development of a clod having an initial internal state $\Gamma$ swelling in a limited volume (case 2) or a non-limited volume (case 1).

$1 / \gamma d:$ volume massique ; mass $/$ volume ratio $\left(\mathrm{cm}^{3} / \mathrm{g}\right)$

$W$ : teneur en eau; water content $\left(\mathrm{g} / \mathrm{g} \mathrm{ou} \mathrm{cm}^{3} / \mathrm{g}\right)$

$V_{S} \quad$ : volume de solide; solid volume $\left(\mathrm{cm}^{3} / \mathrm{g}\right)$

$V e$ : volume d'eau; water volume $\left(\mathrm{cm}^{3} / \mathrm{g}\right)$

$V a$ : volume d'air ; air volume $\left(\mathrm{cm}^{3} / \mathrm{g}\right)$ 
avec maintien de la porosité interagrégats. On trouve de fait même aux très fortes humidités des mottes d'état interne $\Gamma$ (fig. 3) ;

- ou bien son volume apparent est limité (cas 2). Dans ce cas, le gonflement ne pourrait alors se faire qu'au détriment de la porosité interagrégats. Il y aurait remplacement de cette porosité structurale remplie d'air par de la porosité texturale remplie d'eau, coalescence des agrégats et ainsi fabrication d'une motte $\Delta$ dont le point figuratif s'est rapproché de la droite de saturation.

C'est ce processus qui pourrait expliquer les évolutions constatées d'état interne dans l'horizon labouré des parcelles suivies :

- Dans le cas des labours faits sur sol sec (L1 et L2), la forte porosité extra-mottière obtenue après labour autoriserait un gonflement ultérieur des mottes sans contrainte et donc sans dégradation de la porosité structurale intra.

- Dans le cas d'un labour fait après réhumectation du profil (L3), le gonflement ultérieur (les teneurs en eau mesurées pendant l'hiver atteignent $0,48 \mathrm{~g} / \mathrm{g}$ ) se ferait en volume contraint dans les bandes de labour à faible porosité extra-mottière, d'où la dégradation constatée de porosité intra.

Si tel est le cas la limite entre ces 2 situations correspondrait à l'état hydrique pour lequel il n'y a plus fragmentation par le labour. La détermination de cet état hydrique dans les conditions du champ est difficile, le comportement du matériau (plastique ou fragile) étant fonction non seulement de son état hydrique mais aussi de l'énergie appliquée (FAURE, 1981). On sait seulement d'après notre dispositif que cet état hydrique correspond à une teneur en eau inférieure ou égale à l'humidité à la capacité au champ.

La limite inférieure de plasticité (ATTERBERG, 1911), état hydrique pour lequel on passe d'un comportement fragile à un comportement plastique en condition standard de laboratoire et pour une énergie faible, est pour ce matériau proche de la capacité au champ (annexe I). Ceci confirme qu'au champ, compte tenu des plus fortes énergies mises en œuvre, on puisse avoir un comportement plastique pour des teneurs en eau égales ou plus faibles.

A défaut de plus de précision, on a calculé fréquentiellement les dates à partir desquelles le profil est à la capacité au champ. Au moins à partir de cette date un labour serait de type L3.

En supposant qu'à l'issue de la période de déficit hydrique la teneur en eau du sol est proche de Wpf 4,2 , estimée à $0,23 \mathrm{~g} / \mathrm{g}$ (annexe I), la pluie utile $(\mathrm{P}-\mathrm{ETP})$ nécessaire pour amener les 30 premiers $\mathrm{cm}$ à $0,37 \mathrm{~g} / \mathrm{g}$ de teneur en eau serait d'environ $55 \mathrm{~mm}$ (annexe 1) (3). A noter que ce mode de calcul surestime le temps nécessaire à la réhumectation du profil, l'évapotranspiration réelle du sol, si le sol est nu, étant inférieure à l'ETP.

Les données climatiques permettent alors de placer très approximativement les dates à partir desquelles

(3) Pour amener les 80 premiers $\mathrm{cm}$ de sol à cette teneur en eau, très proche de la capacité au champ, il faudrait alors environ $145 \mathrm{~mm}$ de pluie utile. De fait, c'est à peu près pour cette valeur qu'il y a début d'écoulement des drains (COLLECTIF, 1980).

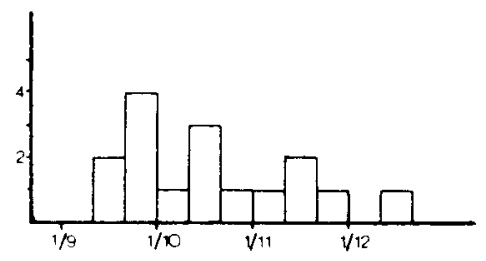

Figure 9

Histogramme des dates à partir desquelles un labour serait de type L3. Années 1967 à 1982.

Estimated dates at which ploughing became L3-type ploughing for years 1967 to 1982 .

un labour serait déjà de type L3 (fig. 9). Il apparaît une forte variabilité interannuelle, avec des risques importants dès la dernière décade de septembre.

\section{CONCLUSION}

Le classement établi et les hypothèses sur lesquelles il se fonde vont au-delà de ce que les contrôles réalisés permettent de conclure. Il s'appuie sur des observations qualitatives faites au champ une année donnée et doit être vérifié. L'intérêt de l'avoir cependant énoncé est double :

- d'une part, il renvoie à des questions relatives au comportement du matériau. Un suivi en laboratoire de l'évolution de l'état interne de mottes soumises à gonflement en volume contraint ou non s'avère nécessaire,

- d'autre part, il suggère les contrôles que devrait nécessairement comporter un dispositif au champ visant à le tester :

- évolution des profils hydriques et de la fissuration dans l'horizon labouré,

- caractérisation des porosités globale et intramottière après labour et de leurs évolutions respectives.

Rappelons que le classement obtenu concerne l'horizon labouré non repris de parcelles travaillées seulement en surface après labour. Il pourrait être différent dans le cas où, après fissuration, une reprise jusqu'au fond de labour était effectuée pour remettre en surface des blocs sous-jacents.

Enfin, le faible effet observé de la fissuration dans l'horizon labouré non repris ne doit pas faire oublier son rôle très important en surface pour la préparation des lits de semence (PELlERIN, 1984). Des gros blocs situés en surface peuvent être progressivement fissurés jusqu'au cœur par alternance d'humectation et de dessiccation, même si les éléments structuraux issus de cette fissuration ne constituent, après aplanissement, qu'un horizon de 5 à $10 \mathrm{~cm}$ d'épaisseur. L'état créé dans l'horizon labouré sous-jacent ne suffit pas à lui seul pour juger d'une date de labour.

Reçu le 24 mai 1985. Accepté le 10 avril 1986.

\section{REMERCIEMENTS}

Nous remercions H. MaNichon (I.N.A.-P.G.) pour l'aide qu'il nous a apportée à l'occasion de ce travail ainsi que le personnel du Domaine expérimental de Saint-Laurent-de-la-Prée. Merci aussi à P. Stengel (I.N.R.A., Avignon) pour ses avis et critiques. 


\section{ANNEXE}

\section{CALCUL DES HUMIDITÉS CARACTÉRISTIQUES}

1 - Teneur en eau à la capacité au champ (Wc) 1971)

$\mathrm{Wpf} 3=0,494 \mathrm{~A}+0,218 \mathrm{Lf}+0,079(\mathrm{Lg}+\mathrm{Sf})+0,08 \mathrm{Sg}$ (OsTY,

Avec A : teneur en argiles (en $\mathrm{g} / \mathrm{g}$ de terre sèche)

Lf : teneur en limons

$\mathrm{Lg}:$ teneur en limons grossiers

Sf : teneur en sables fins

$\mathrm{Sg}$ : teneur en sables grossiers.

Pour $\mathrm{A}=0,60 ; \mathrm{Lf}=0,35 ; \mathrm{Lg}+\mathrm{Sf}=0,035 ; \mathrm{Sg}=0,015$ : $\mathrm{Wpf} 3=0,37 \mathrm{~g} / \mathrm{g}$

Or en sol argileux Wc $=$ Wpf3 (Perigaud, 1963), d'où $\mathrm{Wc}=0,37 \mathrm{~g} / \mathrm{g}$

2 - Teneur en eau au point de flétrissement permanent ( U.pt4,2)

En sol argileux $W p f 4,2=W c / 1,6$ (Perigaud, 1963)

$\mathrm{Wpf} 4,2=0,23 \mathrm{~g} / \mathrm{g}$

\section{3 - Teneur en eau à la limite inférieure de plasticité (W lip)}

$\mathrm{W}$ lip $=0,411 \mathrm{~A}+0,279 \mathrm{Lf}+0,145 \mathrm{~S}+2,9$ (RemY, 1971)

Avec A, Lf, S, teneurs en argiles, limons, sables exprimées en $\mathrm{g} / 100 \mathrm{~g}$ de terre sèche.

W lip $=0,38 \mathrm{~g} / \mathrm{g}$

4 - Hauteur d'eau ( $\mathrm{H}$, en $\mathrm{mm}$ ) nécessaire pour amener les 30 premiers $\mathrm{cm}$ de sol de 0,23 a $0,37 \mathrm{~g} / \mathrm{g}$ d'humidité pondérale

C'est la hauteur d'eau contenue dans la masse de sol occupant les 30 premiers $\mathrm{cm}$ de sol à $0,37 \mathrm{~g} / \mathrm{g}$ de teneur en eau diminuée de la hauteur d'eau contenue dans cette même masse de sol à $0,23 \mathrm{~g} / \mathrm{g} \mathrm{d}$ 'humidité.

En admettant qu'à la capacité au champ le sol a une densité proche de celle des mottes $\Gamma$, on a pour $\mathrm{W}=0,37 \mathrm{~g} / \mathrm{g}, \gamma \mathrm{d} \simeq 1,25$.

La hauteur d'eau contenue dans les 30 premiers $\mathrm{cm}$ de sol à $0,37 \mathrm{~g} / \mathrm{g}$ d'humidité est alors égale à $300 \times 1,25 \times 0,37 \mathrm{~mm}$.

La hauteur d'eau contenue dans la même masse de sol à $0,23 \mathrm{~g} / \mathrm{g}$ d'humidité est égale à $300 \times 1,25 \times 0,23 \mathrm{~mm}$.

D'où $\mathbf{H}=300 \times 1,25(0,37-0,23) \simeq 55 \mathrm{~mm}$.

\section{RÉFÉRENCES BIBLIOGRAPHIQUES}

Atterberg A., 1911. Über die physikalische Bodenuntersüchung. Int. Mitt. für Bodenkunde, 1, 7-9.

Capillon A., Pellerin S., 1984. La maîtrise du travail du sol en Marais poitevin : nouveau point clé de la conduite des exploitations. Bull. Tech. Inf. Min. Agric. Fr., 389, 219-228.

Cardon-Dubois F., 1985. Fissuration à l'humectation d'un sol argileux. Mém. ENITA Dijon-Quetigny, 38 p. + annexes.

Collectif, 1980. Les Marais de l'Ouest. Rapp. DGRST-INRA.SAD (Unité INA-PG), 172 p. + annexes.

Damour L., Nicoulaud J. P., 1975. Tendances nouvelles en préparation des terres argileuses. Bull. Tech. Inf. Min. Agric. Fr., 304305, 687-694

Faure A., 1981. A new conception of the plastic and liquid limits of clay. Soil and Tillage Res., 1, 97-105.

Garreau J., 1976. Reprise des terres lourdes au printemps. L'expérience des Marais de l'Ouest. Entreprises Agricoles, mars 1976.

Haines, 1923. The volume changes associated with variations of water contents in soils. J. Agr. Sci., 13, 296-320.

Henin S., 1976. Cours de physique du sol. 2 vol., ORSTOM - Editest. 159 p. et $221 \mathrm{p}$.

Henin S., Gras R., Monnier G., 1969. Le profil cultural. $2^{\text {e }}$ éd., Masson, Paris, 332 p.

Manichon H., 1982a. Influence des systèmes de culture sur le profil cultural : Elaboration d'une méthode de diagnostic basée sur l'observation morphologique. Thèse de Doct. Ing. INA-PG, 214 p. + annexes.
Manichon H., 1982b. L'action des outils sur le sol : appréciation de leurs effets par la méthode du profil cultural. Sci. Sol, 3, 203-219.

Mengel D. B., Barber S. A., 1974. Development and distribution of the corn root system under field conditions. Agron. J., 66, 3, 341344.

Morizet J., Dejou J., Guyot J., 1970. Principales caractéristiques physico-chimiques des sols de type vertique et sodique dans le Marais rochefortais à Saint-Laurent-de-la-Prée (Charente-Maritime). Sci. Sol, 5, 11-24.

Nijs R., 1978. Les sols du Marais poitevin. Ann. Soc. Sci. Nat. Charente-Maritime, 6 (5), 398-424.

Osty P. L., 1971. Influence des constituants du sol sur son humidité à pF3. Ann. Agron., 22 (4), 451-476.

Pellerin S., 1984. Etude des pratiques de travail du sol dans les exploitations du Marais poitevin. Thèse Doct. Ing. INA-PG, 165 p. + annexes.

Perigaud S., 1963. Contribution agronomique à la mise en valeur de la Brenne (sols hydromorphes). Ann. agron., 14, 3, 261-377.

Remy J. C., 1971. Influence de la constitution physique des sols sur leur comportement mécanique ; signification des limites d'Atterberg en matière de travail du sol. Ann. agron., 22, 3, 267-290.

Tardieu F., 1984. Etude au champ de l'enracinement du maïs. Influence de l'état structural sur la répartition des racines, conséquences sur l'alimentation hydrique. Thèse Doct. Ing. INA-PG, 232 p. + annexes. 